\title{
Perfil epidemiológico das pacientes ginecológicas do ambulatório escola de Franca: uma visão e análise de prontuários eletrônicos
}

\author{
Epidemiological profile of gynecological patients at the Franca school outpatient clinic: a \\ view and analysis of electronic records \\ Perfil epidemiológico de los pacientes ginecológicos en la clínica ambulatoria de la \\ escuela Franca: una visión y análisis de los registros electrónicos
}

Felipe Sant' Maria Naques ${ }^{1 *}$, Jeferson Elias Vargas Rezende ${ }^{1}$, Vivian Zampieri de Souza ${ }^{1}$, Elisabete Lilian Dair ${ }^{1}$, Marcelo Ballaben Carloni'.

\section{RESUMO}

Objetivo: Traçar perfil epidemiológico das pacientes ginecológicas do Ambulatório Escola do curso de Medicina da Universidade de Franca (Unifran), entre 2015 e 2018. Métodos: Foram avaliados os prontuários dos atendimentos em ginecologia do Ambulatório escola da Unifran no período descrito, sendo incluídos os que se apresentavam completos os dados: idade, peso, altura, índice de massa corporal (IMC), frequência cardíaca (FC), queixa principal (QP), antecedentes pessoais, paridade (GPAC) e uso de métodos anticoncepcionais. Sendo excluídos prontuários incompletos. Analisamos os dados no programa estatístico STATA versão 11. Resultados: O perfil epidemiológico encontrado foi: média etária de 43,9 anos, média de 2,4 gestações, paridade de 2,15, cesáreas de 0,81, abortos de 0,27 e IMC 28,38. De acordo com a faixa etária avaliada, (subdividida em 4 grupos), encontrou-se predomínio de uma QP, comorbidade e método contraceptivo em uso. Dessa forma, a prevalência de queixas principais foram alterações de ciclo menstrual (32\%), 86\% não apresentaram comorbidades nos antecedentes pessoais e o uso de anticoncepcionais combinados orais $(27,5 \%)$ superaram outros métodos. Conclusão: O estudo proporcionou traçar o perfil epidemiológico das pacientes, assim como as principais patologias ginecológicas levam a procurar consultas médicas. No entanto, falta de dados completos levou a exclusão de um grande número de prontuários.

Palavras-chave: Doenças dos genitais femininos, Saúde da mulher, Perfil epidemiológico, Ambulatórios, Doenças ginecológicas.

\begin{abstract}
Objective: To draw up an epidemiological profile of gynecology patients at the Unifran School Outpatient Clinic from 2015 to 2018. Methods: The data were collected retrospectively from all the consultations that had the data to be analyzed, of which: age, weight, height, body mass index (BMI), heart rate (HR), main complaint (QP), antecedents people and parity (GPAC). The data analysis was performed using STATA statistical program version 11. Results: The profile found was of a 43.9 year old patient, number of pregnancies 2.4, parity 2.15, cesarean sections 0.81 , abortions 0.27 , with BMI 28.38 . According to the age group evaluated, (subdivided into 4 groups), there was a predominance of a QP, comorbidity and contraceptive method in use. In addition, the main complaints were changes in menstrual cycle (32\%) and about $86 \%$ had no comorbidities, and combined oral contraceptive use (27.5\%) outperformed other methods. Conclusion: The study provided a profile of patients, as well as the main pathologies that demand doctors. However, the lack of complete data made it difficult to survey more patients.
\end{abstract}

Keywords: Females genital diseases, Women's health, Epidemiological profile, Ambulatories, Gynecological diseases.

${ }^{1}$ Universidade de Franca (UNIFRAN), Franca - SP. *E-mail: felipenaques@gmail.com 


\section{RESUMEN}

Objetivo: Elaborar un perfil epidemiológico de los pacientes ginecológicos de la Clínica de Pacientes Externos de la Escuela Unifran de 2015 a 2018. Métodos: Los datos fueron recogidos retrospectivamente de todas las consultas que tenían los datos para ser analizados, de los cuales: edad, peso, altura, índice de masa corporal (IMC), ritmo cardíaco (RH), queja principal (QP), antecedentes de las personas y paridad (GPAC). El análisis de los datos se realizó utilizando el programa estadístico STATA versión 11. Resultados: El perfil encontrado fue de una paciente de 43,9 años, número de embarazos 2,4, paridad 2,15, cesáreas 0,81 , abortos 0,27 , con un IMC de 28,38. Según el grupo de edad evaluado, (subdividido en 4 grupos), existía un predominio de una $\mathrm{QP}$, comorbilidad y anticonceptivo en uso. Además, las principales quejas eran los cambios en el ciclo menstrual (32\%) y alrededor del $86 \%$ no tenía comorbilidades, y el uso de anticonceptivos orales combinados $(27,5 \%)$ superaba a los otros métodos. Conclusión: El estudio proporcionó un perfil de los pacientes, así como las principales patologías que son buscadas por los médicos. Sin embargo, la falta de datos completos dificultó la realización de encuestas a más pacientes.

Palabras clave: Enfermedades genitales femeninas, Salud de la mujer, Perfil epidemiológico, Ambulatorios, Enfermedades ginecológicas.

\section{INTRODUÇÃO}

No Brasil, nas últimas décadas, vem se ampliando o interesse em utilizar bancos de dados originados de forma rotineira pelos serviços de saúde como ferramenta na elaboração de políticas de saúde e no planejamento e gestão dos mesmos (BITTENCOURT AS, et al., 2005). Através dessa análise pode-se detectar o perfil epidemiológico dos pacientes atendidos nas diversas áreas da saúde com o objetivo de facilitar, dinamizar o atendimento e auxiliar diagnósticos futuros.

Em 2004, a Política Nacional de Assistência Integral à Saúde da Mulher (PNAISM) foi criado com foco na promoção de saúde e garantia dos direitos das mulheres (FREITAS GL, et al., 2009). Esse programa trouxe diversos benefícios, entre eles acesso integral aos cuidados de saúde voltado às mulheres, que a partir de então passou a ser assegurado por lei.

Dessa forma, garantindo o grande acesso das usuárias ao SUS (Sistema Único de Saúde), decorrente da diversa gama de atendimentos oferecidos à mulher, sendo esses desde pré-natal, prevenção de câncer de mama e de colo de útero até o atendimento ambulatorial relacionado as mais variadas queixas, entre elas, climatério, infecções sexualmente transmissíveis (ISTs), menarca e sangramento uterino anormal (VIEGA SMF e PENNA CMM, 2013).

O conhecimento do perfil do paciente que está sendo atendido possibilita melhor direcionamento das verbas do Sistema Único de Saúde, otimizando o atendimento, uma vez que essas serão destinadas para materiais e profissionais de acordo com a demanda local (BITTENCOURT AS, et al., 2005).

Existe no Brasil uma dificuldade de acesso à consultas ginecológicas na atenção básica de saúde, uma vez que o número de profissionais ginecologistas não consegue suprir a demanda de atendimento. Sendo assim, as consultas são agendadas após um longo tempo de espera. Dessa forma, muitas pacientes acabam por negligenciar suas queixas e buscam atendimento médico apenas nas portas de entrada diretas, ou seja, em Pronto Socorros (PS) e Unidades de Pronto Atendimento (UPAs), quando seus quadros estão agudizados e agravados. Portanto, a prevenção e promoção de saúde está deficitária por falta de profissionais atendendo na Rede Pública (MINISTÉRIO DA SAÚDE, 2002; BEGA AG, et al., 2017).

Tradicionalmente, o cuidado com a saúde é focado principalmente nas crianças e nas mulheres apenas em relação ao seu aspecto reprodutivo, não se organizando para atender e acolher as demandas femininas fora do período reprodutivo e as demandas masculinas. Assim como não existem muitos trabalhos que façam uma análise da morbimortalidade separada dos sexos, sendo comum encontrá-los numa abordagem geral, também não há muitos que avaliam o perfil epidemiológico das pacientes atendidas em consulta de ginecologia e suas queixas prevalentes (GOMESA KRO e D'A TANAKA AC, 2003; MACHIN R, et al., 2011). 
O ambulatório de ginecologia da faculdade de Medicina da Unifran atende uma grande quantidade de mulheres com um abrangente número de patologias que varia sua prevalência de acordo com a idade e até mesmo dentro de uma determinada faixa etária. Esse fato torna importante conseguir traçar o perfil epidemiológico das pacientes e encontrar as queixas mais prevalentes, a fim de direcionar os atendimentos e auxiliar o serviço de saúde local na sua gestão.

Este estudo teve como objetivo identificar o perfil epidemiológico das pacientes atendidas no ambulatório escola de ginecologia de Franca-SP, por alunos do quarto ano, entre o período de 2015 a 2018, a fim de detectar o tipo de paciente mais atendida, para assim poder traçar metas para possível melhoria nos atendimentos.

\section{MÉTODOS}

Trata-se de um estudo retrospectivo com avaliação de 3.298 prontuários eletrônicos das pacientes atendidas na especialidade de ginecologia do Ambulatório Escol da faculdade de Medicina da Unifran em Franca-SP, com idade entre 12 e 62 anos, nos períodos de janeiro de 2015 a novembro de 2018, gerenciados pelo sistema Tasy (Software de prontuário eletrônico, com certificação pela Sociedade Brasileira de Informática em Saúde).

Foram incluídos no estudo, prontuários eletrônicos com os seguintes dados preenchidos por completo: Idade, peso, altura, índice de massa corpórea (IMC), frequência cardíaca (FC), queixa principal (QP), antecedentes pessoais, paridade (GPAC) e método anticoncepcional. Foi excluído do estudo todo prontuário eletrônico com ausência de qualquer dado citado anteriormente. Obteve-se autorização para realização da pesquisa pelo Comitê de Ética em Pesquisa da Santa Casa de Franca, conforme parecer número 3.398.891, de acordo com o Conselho Nacional de Saúde, resolução 466/12, que visa regulamentar pesquisa envolvendo seres humanos e sob a autorização da direção clínica do hospital para acesso aos prontuários.

Os dados foram analisados com ajuda do programa estatístico STATA versão 11 (StataCorp LP, Texas, USA), com estratificação dos pacientes conforme os critérios pré-definidos e colocados em gráficos para melhor visualização.

\section{RESULTADOS}

Incluiu-se na análise do presente trabalho 432 prontuários eletrônicos de diferentes pacientes, com exclusão de 2.866 prontuários por não abarcarem os critérios de inclusão em sua totalidade.

Subdividimos o grupo total de pacientes em 4 subgrupos: Grupo 1: Adolescentes (idade entre 12-19 anos), Grupo 2: Adultas Jovens (idade entre 20-40 anos); Grupo 3: Adultas (idade entre 41-59 anos); e Grupo 4: Idosas (idade maior ou igual 60 anos). A partir desses subgrupos analisamos as queixas principais, as comorbidades e métodos contraceptivos de cada um deles. Foi calculado o perfil epidemiológico da paciente atendida na Ambulatório Escola de Franca, através das seguintes médias: idade de 43,9 anos (+/-15,02), número de gestações de $2,4(+/-2)$, paridade de $2,15(+/-1,82)$, cesáreas por paciente $0,81(+/-1,13)$, abortos por paciente $0,27(+/-0,57)$ e IMC 28,38 (+/- 6,65).

Ao iniciar a coleta de dados deparamos com problemas referentes à inserção de pacientes na pesquisa, uma vez que faltavam dados e assim não pudemos incluí-los; sendo que $86,9 \%$ dos prontuários não foram incluídos por não preencherem todos os quesitos para a análise.

O G1 teve como queixas principais abordadas: alterações do ciclo menstrual (32\%), exame de rotina $(18 \%)$, dor abdominal (18\%), outras queixas (32\%). A maioria das pacientes desse grupo não apresentaram comorbidades (86,5\%) tendo apresentado asma em $4,5 \%$, anemia $4,5 \%$, enxaqueca $4,5 \%$, Síndrome dos ovários policísticos (SOP) 4,5\%. Quanto aos métodos contraceptivos, a maioria não usava (63,5\%), seguido pelo uso de anticoncepcional oral (27,5\%) e métodos de barreira (9\%).

No G2 encontramos como queixa principal mais prevalente o exame de rotina (33\%) seguido de dor abdominal (16,5\%), alterações do ciclo menstrual (15\%), planejamento familiar ( $8 \%)$, leucorreia $(6 \%)$, coleta 
de exame de papanicolau (3,5\%), alterações nas mamas (3,5\%), sangramento uterino anormal (2\%), SOP $(1,5 \%)$, outros $(11 \%)$. Nesse grupo também houve uma predominância do gupo sem nenhuma comorbidade (75\%) seguidos de hipertensão arterial sistêmica (8,5\%), depressão (4\%), diabetes mellitus 2 (DM2) (3,5\%), $\operatorname{SOP}(2,5 \%)$, hipotireoidismo $2,5 \%$ e outros $(7,5 \%)$, visuallizadas no Gráfico 1. Nessa faixa etária $38 \%$ não utilizavam métodos contraceptivos. Dentre as pacientes praticantes de contracepção, os métodos mais utilizados foram o anticoncepcional oral $(34,5 \%)$, métodos de barreira $(12,5 \%)$, injetável combinado $(5,5 \%)$, laqueadura $(5,5 \%)$, progestágeno via oral $(2 \%)$, parceiro com vasectomia $(1,5 \%)$ e coito interrompido $(0,5 \%)$, apresentados no Gráfico 2.

Gráfico 1 - Prevalência de comorbidades em pacientes com idade entre 20 e 40 anos.

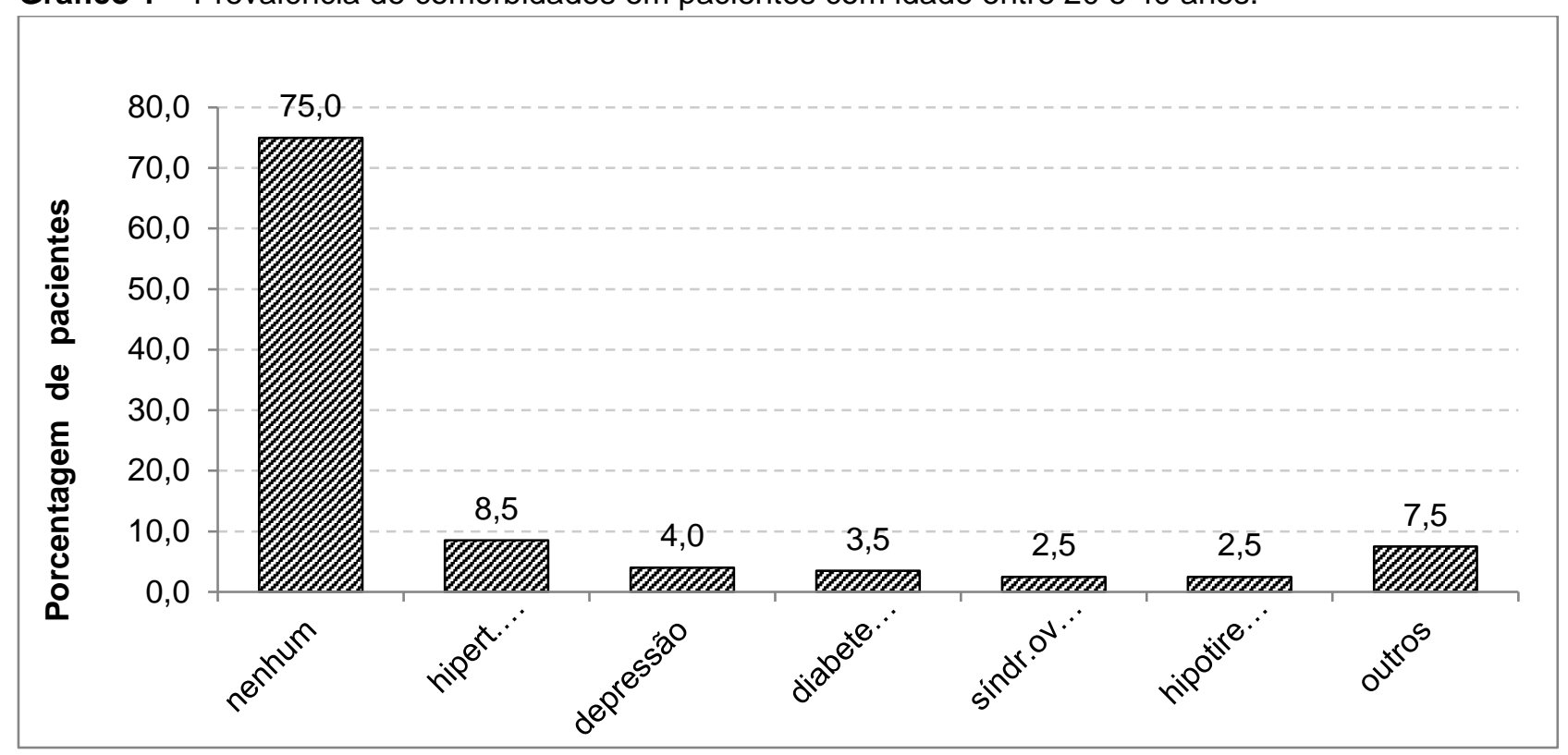

Fonte: Naques FSM, et al., 2020.

Gráfico 2 - Prevalência de métodos contraceptivos em pacientes com idade entre 20 e 40 anos.

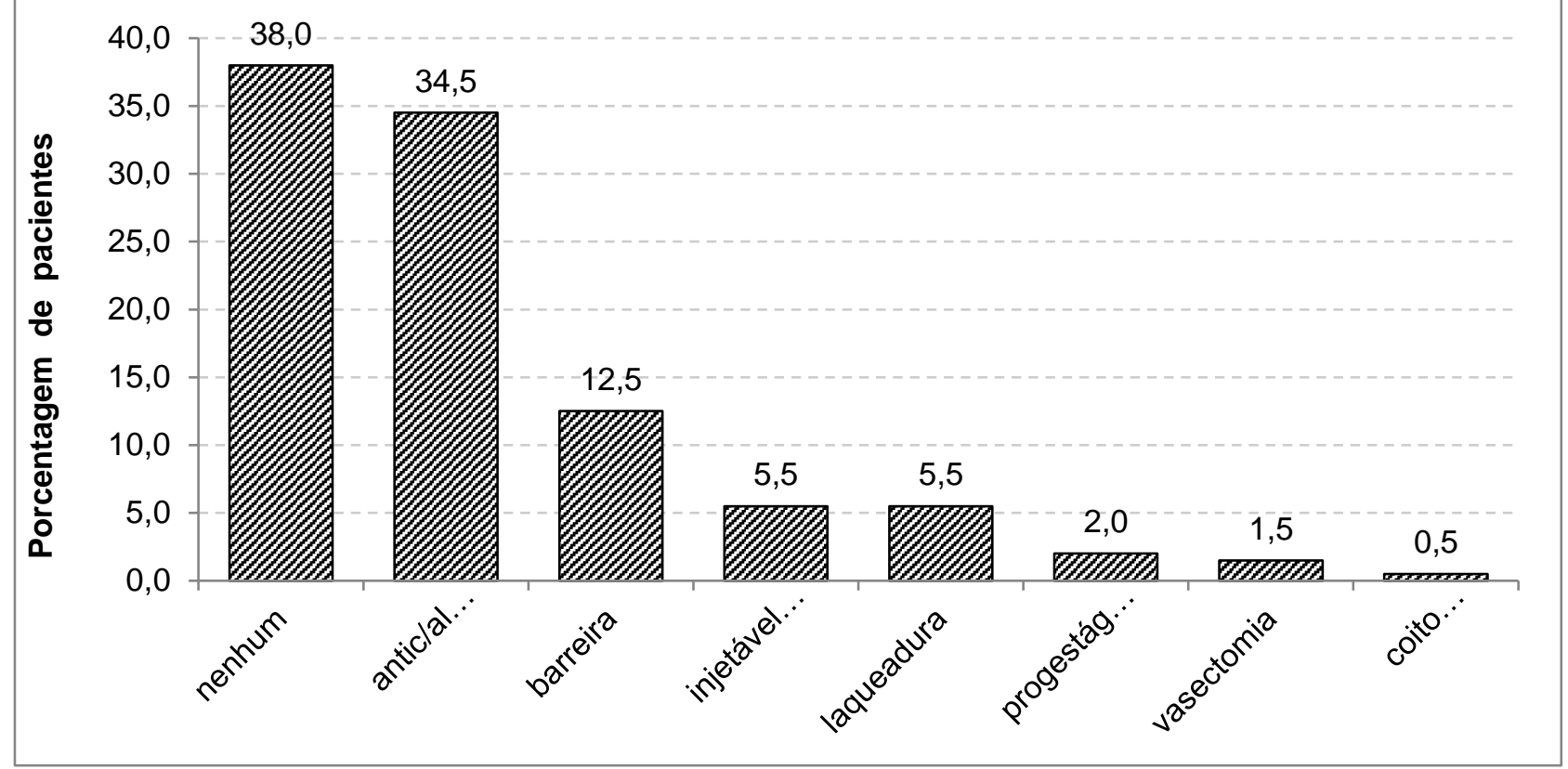

Fonte: Naques FSM, et al., 2020. 
No grupo G3 as queixas principais mais prevalentes foram exame de rotina (47,5\%), dor abdominal $(14,5 \%)$, alterações do ciclo menstrual $(7,5 \%)$, climatério (4\%), leucorreia $(3,5 \%)$, alterações das mamas (3\%), realização de Papanicolau (2,5\%), sangramento uterino anormal $(2,5 \%)$ e outros (15\%). Dessas, $47 \%$ não apresentaram comorbidades presentes no Gráfico 3, sendo a prevalência de hipertensão arterial sistêmica de $33 \%$, DM2 $14,5 \%$, hipotireoidismo $9 \%$, depressão $3 \%$, SOP $2,5 \%$ e outras $21 \%$.

Gráfico 3 - Prevalência de comorbidades em pacientes com idade entre 41 e 59 anos.

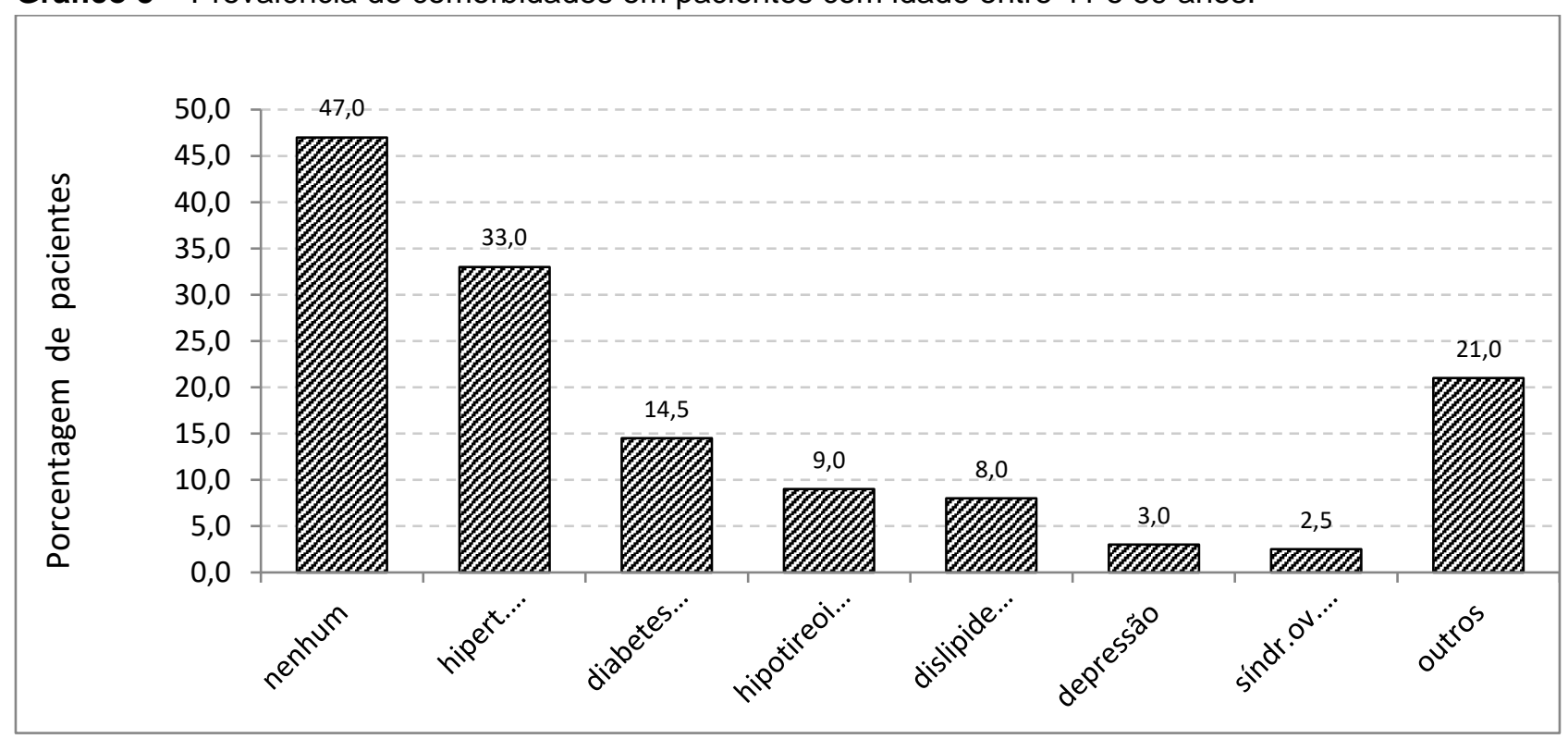

Fonte: Naques FSM., 2020.

Cinquenta e cinco por cento dessas não usavam métodos contraceptivos, $13,5 \%$ usavam anticoncepcional oral, $13,5 \%$ tinham sido submetidas a laqueadura tubárea, 11,5\% usavam métodos de barreira 11,5\%, 2,5\% eram histerectomizadas, $1,5 \%$ usavam progestágeno via oral, $1 \%$ tinham parceiros com vasectomia, $0,5 \%$ usavam anticoncepção injetável combinada e 0,5\% eram usuárias de DIU, dados visíveis no Gráfico 4.

Grupo 4 - Prevalência de métodos contraceptivos em pacientes com idade entre 41 e 59 anos.

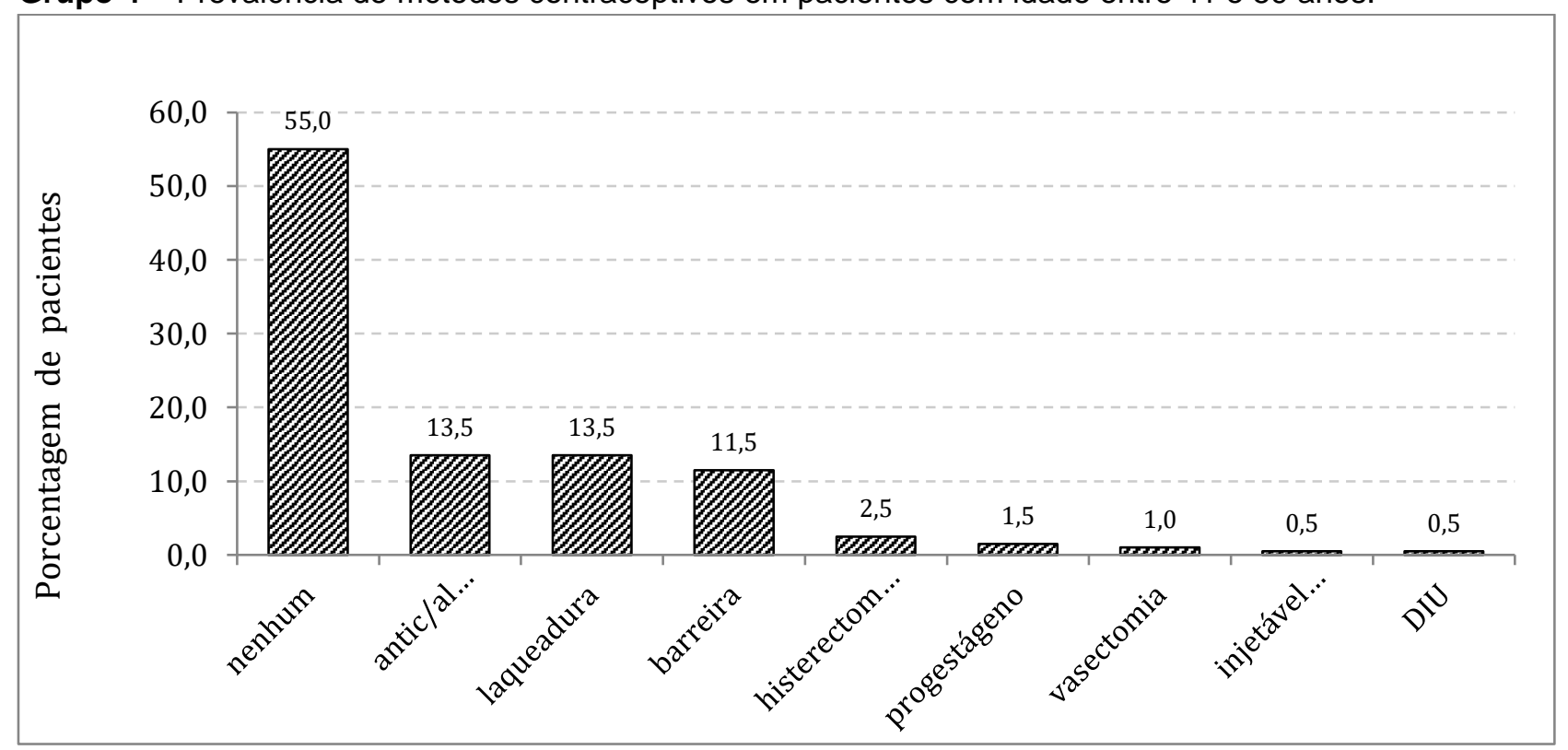

Fonte: Naques FSM, et al., 2020. 
A distribuição das queixas principais no grupo G4 ficou da seguinte forma: exame de rotina $(65,5 \%)$, dor abdominal $(8 \%)$, alterações das mamas $(5,5 \%)$, leucorreia $(3 \%)$ e outros $(18 \%)$. Nesse grupo $(30 \%)$ das pacientes não apresentaram comorbidades, as que apresentaram eram divididas em hipertensão arterial sistêmica (61,5\%), DM2 (24,5\%), hipotireoidismo (7\%), depressão (5,5\%), antecedente de SOP (2,5\%) e outros (9,5\%), apresentados no Gráfico 5.

Gráfico 5 - Prevalência de comorbidades em pacientes com idade maior ou igual 60 anos.

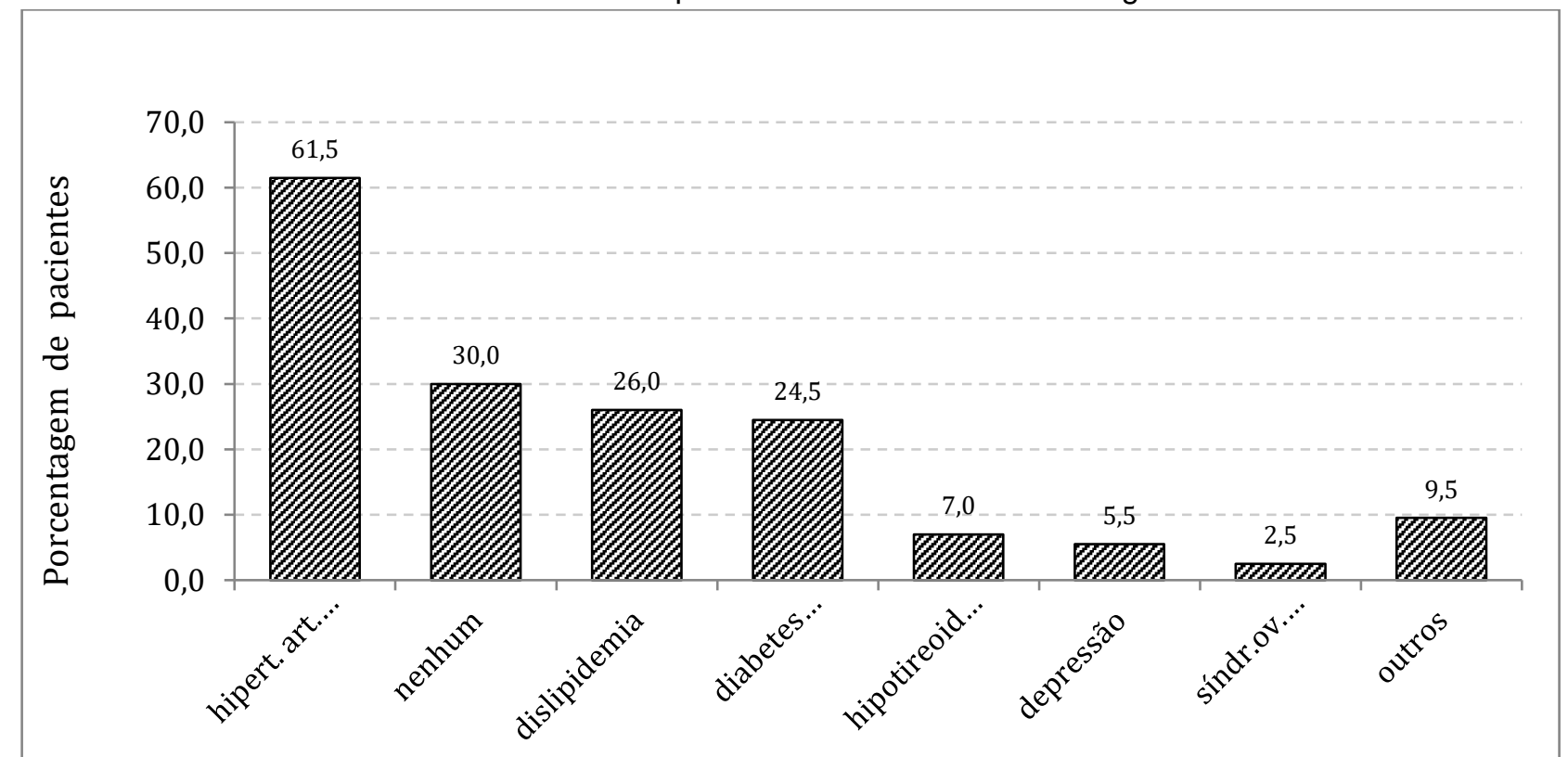

Fonte: Naques FSM, et al., 2020.

Dentre as pacientes desse grupo, apesar de não estarem em idade reprodutiva, pesquisamos métodos definitivos prévios de contracepção, sendo eles histerectomia $(5,5 \%)$ e laqueadura tubárea (16,5\%), sendo assim $78 \%$ não foram submetidas a métodos permanentes. Pesquisou-se também o uso de preservativo e não houve relato de uso, dados observados no Gráfico 6.

Gráfico 6 - Prevalência de métodos contraceptivos em pacientes com idade maior ou igual 60 anos.

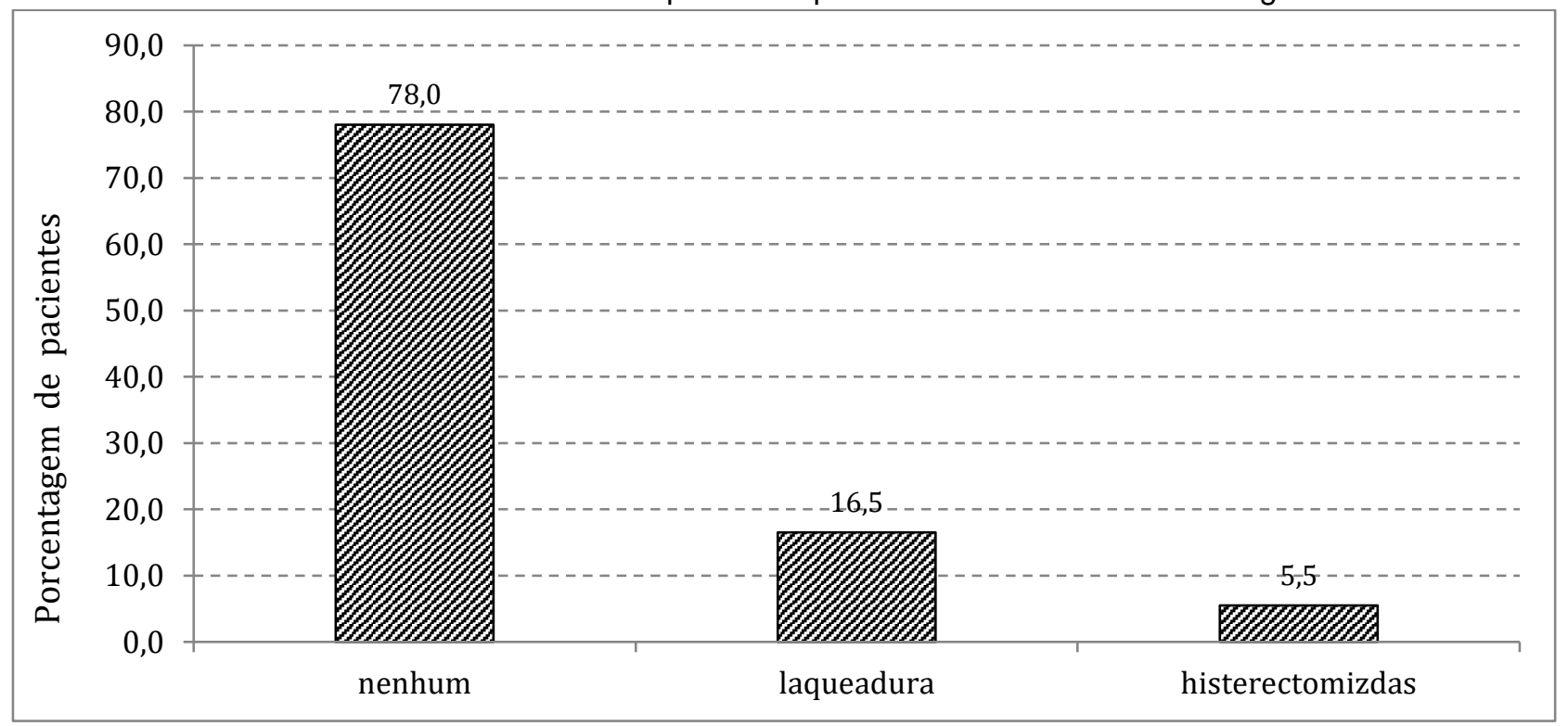

Fonte: Naques FSM, et al., 2020. 


\section{DISCUSSÃO}

Os achados da presente pesquisa apontam um perfil de pacientes com maior frequência de comorbidades diretamente proporcional a idade, com menor uso de métodos contraceptivos em idades fora do período reprodutivo e nenhuma paciente em uso de método de barreira após 60 anos. Além disso, o grupo 3 com idade entre 41-59 anos, foi o que mais procurou atendimentos em razão de exames de rotina, refletindo a eficiência e empoderamento da mulher. O objetivo do trabalho é apontar as principais demandas e facilitar os diagnósticos e atendimentos futuros. Ademais, possibilita 0 melhor direcionamento dos recursos e consequentemente, otimização dos atendimentos.

Nos últimos anos ficou evidente o aumento da prevalência de doenças crônicas como diabetes, hipertensão, depressão e outras. Atualmente, as condições crônicas são responsáveis por $60 \%$ do ônus decorrente das doenças no mundo, isso condiz com o envelhecimento populacional que está ocorrendo (ROSSY ISP, et al., 2011).

Durante o delineamento do estudo surgiram problemas no critério de inclusão das pacientes, pois faltavam dados no prontuário eletrônico, apesar desses dados estarem presentes na consulta médica anotados em uma ficha de avaliação, no entanto, não foram escritos na plataforma TASY. Sendo assim, cerca de $86,9 \%$ dos prontuários foram excluídos. A despeito dessa dificuldade, surgiu a resolução do problema após a coleta dos dados, onde foi inserida na plataforma TASY um campo específico para colocar peso, altura, pressão arterial, frequência cardíaca e saturação.

Ao analisarmos as pacientes de acordo com os grupos separados pelas faixas etárias, encontramos que quanto maior a idade, maior o número de comorbidades, entre elas hipertensão arterial sistêmica e DM2. Essas se encaixam nas doenças crônicas que poderiam na maior parte das vezes ser evitada e com complicações preveníveis com medidas comportamentais, fazendo uma medicina mais preventiva, incentivando o estilo de vida saudável. As estratégias para minimizar o surgimento das condições crônicas, suas complicações e a mortalidade decorrentes dessas incluem deteç̧ão precoce, aumento da prática de atividade física, redução do tabagismo e restrição do consumo excessivo de alimentos não saudáveis (OMS, 2003; OMS 2015).

Ao obter-se como média um IMC de $(28,38)$ nas pacientes, oberservamos uma predominância de sobrepeso nas pacientes estudadas, em concomitância com o aumento de peso observado mudialmente. Tal achado implica em várias doenças crônicas, como Diabetes Melitus, Hipertensão Arterial, Dislipidemias, dentre outras. ( MALACHIAS MVB, et al, 2016) Associado ao elevado IMC, foi observado, em todas as faixas etárias do estudo, a Hipertensão Arterial como principal comorbidade. Dessa forma, demonstrando a associação na prática entre sobrepeso e Hipertensão arterial; isso nos leva a reforçar a importância da saúde pública em enfrentar o aumento de peso populacional. ( MALACHIAS MVB, et al, 2016).

Dessa forma, cabe aos funcionários da saúde orientar e incentivar tais ações afim de prevenir as doenças crônicas que cada vez mais atingem a população, e consequentemente diminuir os gastos com saúde pública nas gerações futuras.

Outro fato relevante no contexto atual é a necessidade de uma consulta humanizada e completa, com o objetivo de atender as várias demandas das pacientes. Para muitas mulheres, os ginecologistas são encarados como especialistas no sexo feminino para realização de exames de rotina e abordagem dos mais diversos sintomas apresentados de origem ginecológica ou não. Portanto, a consulta ginecológica pode ser um momento oportuno para investigar, diagnosticar e tratar comorbidades não ginecológicas presentes, não necessitando, em grande parte das vezes, do amparo de outros profissionais especialistas (clínicos, endocrinologistas, cardiologistas, entre outros) que podem ser de difícil acesso. Dessa forma, os ginecologistas têm a oportunidade de prevenir e tratar uma grande variedade de doenças (CUNNINGHAM FG e LEVENO KJ, 2010).

A consulta ginecológica deve ser oportuna, cabendo ao médico orientar como melhorar a alimentação: reduzindo a ingesta de açúcares refinados e carboidratos com baixo índice glicêmico, reduzir a ingesta de sal ( $<5 \mathrm{~g} / \mathrm{dia})$. Estimular o aumento de fibras e proteínas, nutrientes sacietógenos, decrescendo a ingestão 
energética; afim de controlar o ganho de peso desenfreado na população, que cada vez está mais obesa. Vale salientar uma ingesta hídrica adequada, $30 \mathrm{ml} / \mathrm{kg} / \mathrm{dia}$, otimizando o funcionamento intestinal (WORLD HEALTH ORGANIZATION, 2015). Além de incentivar a prática de atividade física, cerca de 150 minutos semanais. (MINISTÉRIO DA SAÚDE, 2017).

Ainda é grande a população com acesso limitado à assistência médica, sobretudo quando se trata do segmento mais carente (SENNA MCM, 2002). Desse modo, o atendimento deve ser integral e global, atendendo todas as necessidades da paciente (FREITAS GL, 2009).

Além disso, encontramos que quanto maior a idade dentro do período fértil, mais variável é o método contraceptivo utilizado sendo no G1 apenas anticoncepcional oral e métodos de barreira, no G2 anticoncepcional oral, barreira, injetável combinado, laqueadura, progestágeno, vasectomia e coito interrompido e no G3 anticoncepcional oral, laqueadura, barreira, histerectomia, progestágeno, vasectomia, injetável combinado e DIU. Já no G4 como são mulheres que não estão mais em idade reprodutiva, têm uma menor a preocupação do uso de preservativos. Porém, a função do preservativo vai muito além da contracepção, sendo um importante método para a prevenção de DST, e a população mais idosa cada vez mais negligencia seu uso, dado nitidamente encontrado nas pacientes do nosso estudo.

A melhora da qualidade de vida, aliado aos avanços tecnológicos em saúde, como os tratamentos de terapia hormonal e medicações para impotência, têm permitido o descobrimento de novas experiências, como o sexo entre os idosos. No entanto, a ocorrência de práticas sexuais desprotegidas contribui para que essa população se torne vulnerável às infecções pelo HIV e outras doenças sexualmente transmisíveis, como sífilis, clamídia e gonorreia (LAROQUE MF, et al., 2011). Esse comportamento de risco, encontrado na população analisada, corrobora com os achados do Ministério da Saúde que constata o aumento do número de casos de IST em idosos (MINISTÉRIO DA SAÚDE, 2011).

Enquanto no G1 a queixa principal mais prevalente foi as alterações do ciclo menstrual (32\%), nos G2 e G3 prevaleceram a busca por exames de rotina, 33 e $47 \%$ respectivamente. Dessa forma podemos observar, que quanto maior a idade da paciente, maior a conscientização da importância do exame ginecológico de rotina. Hábito esse que culturalmente conseguiu ser implantado nas pacientes atendidas por essa unidade de saúde na cidade de Franca-SP, devido à promoção de saúde proporcionada através do ensino em saúde pelos alunos que atendem no Ambulatório Escola da UNIFRAN.

A consulta médica, de modo geral, tem grande impacto na vida dos pacientes. No caso das mulheres, a consuta ginecológica é fundamental para prevenção do câncer, especialmente de colo do útero e o de mama, sendo também indispensável no planejamento familiar, pré-natal, atendimento a patologias obstétricas e controle de infecções sexualmente transmissíveis. Assim, vale ressaltar a importância de um bom vínculo com a paciente levando-a a se sentir confortável durante a consulta proporcionando a procura de atendimento sempre que necessário e não ficando apreensiva em relação às consultas (SIMÃO AB, et al., 2004).

O câncer de mama é o tipo mais comum de câncer entre as mulheres no mundo e no Brasil, depois do de pele não melanoma e corresponde a $1 / 4$ dos casos novos a cada ano. Porém, na região Norte do pais prevalece o câncer do colo do útero, que fica em segundo lugar no restante do país (INCA, 2019).

O mesmo pode ser detectado em fases iniciais o que aumenta a possibilidade de tratamentos com menor morbimortalidade. O Ministério da Saúde recomenda que a mamografia de rastreamento seja ofertada para mulheres entre 50 e 69 anos, a cada dois anos (INCA, 2018). Ademais, o câncer do colo do útero com a detecção precoce é uma estratégia para encontrar lesões precursoras ou tumores numa fase inicial e, assim, possibilitar maior chance de cura (INCA, 2019).

O rastreamento abrangente e constante é uma ferramenta eficiente para redução da mortalidade por câncer e estudos mostram que pode reduzir em mais de 70\%, o número de mortes em decorrência ao câncer do colo do útero quando comparado às populações não assistidas. Existem dois motivos para este efeito: 0 primeiro é que na maioria das mulheres é detectado ainda como doença pré-invasora; o segundo é, quando a doença é detectada em sua forma invasora, esta tende a ser diagnosticada em estádios mais precoces, nos quais as chances de cura são muito maiores (GUY JGP, et al, 2015). 
Outro dado alarmante em nosso estudo é o excesso de peso encontrado nas pacientes, fato que corrobora com outros estudos e que parece representar a realidade da população do país. Em âmbito mundial, entre 1980 e 2014, a proporção de obesos mais que dobrou. A elevação da prevalência de obesidade encontra como base mudanças comportamentais ocorridas nas últimas décadas, sobretudo devido à alimentação inadequada e ao sedentarismo (WORLD HEALTH ORGANIZATION, 2014). Nos países de renda média, os sistemas de vigilância têm encontrado tendências de aumento de obesidade (WORLD HEALTH ORGANIZATION, 2014).

A obesidade é causa de redução da qualidade de vida, de incapacidade funcional, redução da expectativa de vida e aumento da mortalidade. Comorbidades como doença renal, osteartrose, câncer, DM2, hipertensão, apneia do sono, doença hepática não alcoólica e, mais importante, doença cardiovascular, estão relacionadas diretamente com o excesso de peso (ABESO, 2011).

Além disso, o sobrepeso e a obesidade são apontados como a segunda causa evitável de câncer, atrás apenas do tabagismo. Ambos estão associados ao aumento do risco de câncer de mama, cólon, endométrio, vesícula, esôfago, pâncreas e rim (INCA, 2006). Por isso, estratégias devem ser propostas com direcionamento de medidas para prevenção do sobrepeso e da obesidade, diminuindo assim o risco de diversas doenças crônicas de forma a diminuir a demanda futura por serviços de saúde, e desta forma, direcionar recursos para outras áreas.

\section{CONCLUSÃO}

A partir do perfil epidemiológico traçado no estudo possibilitou projetar estratégias de ensino para os alunos acerca de principais queixas e a necessidade de maior atenção à anamnese e exame físico para sanar as queixas da população. Além disso, com a coleta de dados foram observadas falhas nas anotações de dados básicos dos pacientes, como peso, altura, IMC, frequência cardíaca, dentre outros. Esse fato auxilia na correção de futuras distorções que poderiam ocorrer devido a dados básicos não estarem anotados. Foi evidenciado, o maior uso de métodos contraceptivos entre o público adulto jovem, porém, as mulheres com idade maior que 60 anos responderam de forma unânime a não aderência ao uso do preservativo. Revelouse excesso de peso na população avaliada no presente estudo.

\section{AGRADECIMENTOS}

Agradecemos à Santa Casa de Franca e a UNIFRAN por nos proporcionar a possibilidade de colher os dados nos nossos horários livres. Assim como agradecemos a nossa co-orientadora por nos mostrar a necessidade do perfil epidemiológico e nos incentivar a fazer a pesquisa científica.

\section{REFERÊNCIAS}

1. BITTENCOURT SA, et al. O Sistema de Informação Hospitalar e sua aplicação na saúde coletiva, Carde de Saúde Pública, Escola Nacional de Saúde Pública Sergio Arouca, Fundação Oswaldo Cruz, Rio de Janeiro, Brasil. 2005.

2. FREITAS GL, et al. Discutindo a política de atenção à saúde da mulher no contexto da promoção da saúde. Rev eletrônica enferm. 2009; 11(2): 424-8.

3. VIEGAS SMF, PENNA CMM. O SUS é universal, mas vivemos de cotas. Ciênc saúde coletiva. 2013; 18(1): 181 -190.

4. BRASIL. Ministério da Saúde. Portaria n. - 2.048/GM, de 05 de novembro de 2002. Regulamento Técnico de sistemas estaduais de urgência e emergência. Brasília, DF, 2002. Acesso em: 09 de setembro de 2018

5. BEGA AG, et al. A busca de assistência à saúde em serviços de pronto atendimento por mulheres adultas. Rev Fund Care Online. 2017; 9(1): 1-14.

6. MACHIN R, et al. Concepção de gênero, masculinidade e cuidados em saúde: estudo com profissionais de saúde da atenção primária. Ciência e Saúde Coletiva. 2011; 16(11): 4503-4512.

7. GOMESA KRO, D'A TANAKA AC. Morbidade referida e uso dos serviços de saúde por mulheres trabalhadoras, Município de São Paulo. Rev. Saúde Pública. 2003; 37(1).

8. ORGANIZAÇÃO MUNDIAL DE SAÚDE. Cuidados inovadores para condições crônicas: componentes estruturais de ação: relatório mundial. Brasília: Organização Mundial de Saúde; 2003.

9. WORLD HEALTH ORGANIZATION. Noncommunicable diseases Fact Sheet. 2015. 
10. ROSSY ISP, et al. Caracterização da demanda em uma Unidade de Saúde da Família. Rev bras med fam comunidade. Florianópolis. 2011;6(20): 175-81.

11. MALACHIAS MVB, et al. 7 ${ }^{\text {a }}$ Diretriz Brasileira de Hipertensão Arterial. Arquivos Brasileiros de Cardiologia, Rio de Janeiro. 2016; 107(3): 3; 1-104.

12. CUNNINGHAM FG, et al. Obstetrics 23rd edition - McGraw Hill's, 2010 e alterações.

13. WORDL HEALTH ORGANIZATION. Healthy diet. Fact Sheet $n^{\circ} 394$ may 2015.

14. MINISTÉRIO DA SAÚDE. Atividade Física 2017.

15. DIRETRIZ DA SOCIEDADE BRASILEIRA DE DIABETES: 2014-2015/ Sociedade Brasileira de Diabetes; São Paulo: AC Farmaceutica, 2015.

16. SENNA MCM. Equidade e política de saúde: algumas reflexões sobre o Programa Saúde da Família. Caderno de Saúde Pública, Rio de Janeiro, 18 suplemento, p 203-211,2002.

17. MINISTÉRIO DA SAÚDE (BR). Casos de Aids notificados no SINAN segundo sexo e faixa etária por ano de diagnóstico, Brasil, 1980-2010. Bol Epidemiol AIDS DST. 2010 [citado 2011 mar 16];7(1):22.

18. LAROQUE MF, et al. Sexualidade do idoso: comportamento para a prevenção de DST/AIDS. Ves. Gaúcha Enferm 2011; 32(4): 774-780.

19. SIMÃO AB, et al. O recorte raça/cor e a saúde reprodutiva em Belo Horizonte e Recife: uma análise exploratória sobre a realidade de consultas ginecológicas, 2002. ENCONTRO NACIONAL DE ESTUDOS POPULACIONAIS, XIV. Anais Ouro Preto MG, Abep, 2004.

20. INSTITUTO NACIONAL DO CÂNCER. Câncer de mama. 2019.

21. INSTITUTO NACIONAL DO CÂNCER. Síntese de resultados e comentários. 2018.

22. INSTITUTO NACIONAL DO CÂNCER. Câncer do colo do útero. 2019.

23. GUY JGP, et al. The Reach and Health Impacts of the National Breast and Cervical Cancer Early Detection Program Cancer Causes Control, 2015.

24. WORLD HEALTH ORGANIZATION. Global status reporto n noncommunicable diseases 2014. Genebra: Word Health Organization; 2015.

25. ABESO. Associação Brasileira para o Estudo da obesidade e da Síndrome Metabólica. Doenças Desencadeadas ou Agravadas pela Obesidade. 2011.

26. INSTITUTO NACIONAL DO CÂNCER. A situação do câncer no Brasil. 2006. 